\title{
Where Should Hospitalist Programs be Housed?
}

\author{
Thomas McGinn, MD, MPH' and Robert Centor, MD² \\ ${ }^{1}$ Chief of General Internal Medicine, Mount Sinai School of Medicine, Mount Sinai Medical Center, New York, NY, USA; ${ }^{2}$ Division of General \\ Internal Medicine, Associate Dean Huntsville Regional Medical Campus, University Alabama, Birmingham, USA.
}

J Gen Intern Med 23(8):1288-9

DOI: $10.1007 / \mathrm{s} 11606-008-0700-3$

(c) Society of General Internal Medicine 2008

$\mathrm{F}$ landers and colleagues have written an important opinion piece that helps advance the critical dialogue regarding the future organization of academic divisions of general internal medicine (ADGIM). ${ }^{1}$ The authors give a balanced view of the current state of ADGIM and some of the challenges now faced as hospitalist programs rapidly grow. According to Flanders, the demand for hospitalists continues to grow for all hospital types. Several pressures have fueled this rapid growth: shrinking profit margins in the health industry, hospitals' financial need to decrease LOS, increasing quality measures imposed by the federal government and insurers, and the recent development of work-duty regulations, which have stimulated teaching hospitals to create non-housestaff inpatient coverage. The latter factor has pushed academic medical centers to rapidly hire hospitalists, both for patient care and education.

It is important to understand the focus of the discussion and to appreciate that the definition of a hospitalist has varied and changed over the years from any physician who spends two to four months on service to someone who spends more than 5 months on inpatient service and has no outpatient responsibilities. Confusion occurs when trying to define an academic hospitalist and compare and contrast duties and expectations when roles and definitions vary dramatically from institution to institution.

As hospitalist programs in academic medical centers grow, a debate is intensifying: where should these programs be housed? The vast majority of hospitalists are general internists, and most academic hospitalist programs currently reside within divisions of general internal medicine. Should hospitalists develop their own divisions or departments, or should they remain within ADGIM? Flanders and colleagues articulate two arguments for maintaining the status quo. First and foremost, they say, hospitalist programs currently lack adequate academic development and infrastructure, making it impossible for them to build or create a dedicated academic division. This argument is valid if we define "academic" broadly, i.e., encompassing research as well as education.

In 2008, each academic unit should strive to become a "triple threat." ${ }^{2}$ Most academic hospitalist programs have extensive clinical expectations and educational responsibilities, but few have research strength. Because academic hospital medicine is still maturing, most academic hospitalists are assistant professors. Most lack training in educational scholarship and formal clinical research. Moreover the lack of

Published online July 10, 2008 associate professors and full professors makes mentorship challenging. The lack of research experience and infrastructure is exacerbated by NIH budget tightening: even established investigators are struggling to retain funding.

We agree that the lack of academic maturity should influence whether hospitalists should be housed in separate divisions. This, however, implies that if only hospitalist medicine had access to research infrastructure and mentors, then separating it out from DGIM would work. In other words, the authors imply that the only problem is bad timing; everything else supports creation of a separate hospitalist division. This indirectly implies and assumes that geography determines a specialty. We disagree with that premise. Consider cardiology (whether invasive, non-invasive, imaging, EP, etc.) or pulmonary medicine (ICU critical care, asthma care, sleep labs, etc.). In both cases, the argument that geography justifies splitting off a part of division to create new divisions would be analogous to that of hospitalist medicine splitting off from internal medicine. Yet they have not done so. This demonstrates that the problems with a separate division of hospital medicine extend beyond the lack of mentors and research infrastructure that are discussed in the article. Splintering a group and widening a divide between inpatient general internists and outpatient general internists could jeopardize and diffuse training, increase health care costs because of heightened redundancy of administrative support, and, most seriously, threaten quality of care, especially in terms of continuity, in major teaching hospitals. Many academic general internists continue to split their time between inpatient and outpatient medicine. They can manage this because their clinics are in the hospital, where their patients, who tend to be sicker, are frequently admitted. General internists manage this work efficiently and provide high-quality care. Where would they fit in in a split system?

The current hospital focus on decreasing lengths of stay leads to patients leaving hospitals "quicker and sicker." In addition, the growing wave of aging baby boomers has forced general internists in ambulatory care practices, especially those in academic medical centers, to care for more and more complex patients with multiple chronic illnesses, often known by the term "tertiary primary care." The movement toward creating a "medical home," which is being piloted by commercial insurers and Medicare, emphasizes the need for smooth transitions between hospitals and primary care sites. Patients will benefit from inpatient and outpatient internists working closely to expedite transitions of care, potentially reducing length of stay and reducing non-reimbursed readmission of patients not adequately followed after discharge.

Internists have the advantage of a patient perspective rather than a disease perspective. This advantage exists regardless of our practice site. We consider hospitalists, outpatient internists and dual-site internists as all patient-centered physi- 
cians. While we adopt different practice skills, we share a common orientation. We believe that internal medicine training will benefit from the different flavors of general internists working together to define education, clinical practice and research issues. For those reasons, we hope that the majority of academic hospitalists remain in academic divisions of general internal medicine.

Corresponding Author: Thomas McGinn, MD, MPH, Chief of General Internal Medicine, Mount Sinai School of Medicine, Mount
Sinai Medical Center, 17 East 102nd Street, P. O. Box 1087, New York, NY 10029, USA (e-mail: Thomas.mcginn@mountsinai.org).

\section{REFERENCES}

1. Flanders SA, Saint S, McMahon L, Howell JD. Where should hospitalists sit within the academic medical center? J Gen Intern Med. 2008;?:?. 2. Centor RM. Erstwhile triple threat. J Gen Intern Med. 2002;17:572-73. 